\title{
On the Admissible Control operators for Linear and Bilinear Systems and the Favard Spaces
}

\author{
F. Maragh \\ H. Bounit \\ A. Fadili \\ H. Hammouri
}

\begin{abstract}
The objective of this work is to give some relationship between the Favard spaces and the $p$-admissibility (resp. $(p, q)$-admissibility) of unbounded control operators for linear (resp; bilinear) systems in Banach spaces. For linear case, this enables to give a simple identification of the space of the 1-admissible control operators in Banach space and it enables us to extend the result of Weiss [29] (for $p=1$ ) on reflexive Banach spaces to a general situation. This result is applied to boundary control systems. The results obtained for bilinear systems generalize those given in Idrissi [16] and Berrahmoune [2] and are applied to diffusion equations of fractional order time distributed order.
\end{abstract}

\section{Introduction}

This paper studies infinite-dimensional bilinear control systems described by:

$$
\left\{\begin{array}{l}
\dot{x}(t)=A x(t)+u(t) B x(t), \quad t \geq 0 \\
x(0)=x_{0}
\end{array}\right.
$$

where $x(\cdot)$ the state of the system (1.1) takes values in a Banach space $X$ (state space), the unbounded operator $(A, \mathcal{D}(A))$ generates a $C_{0}$-semigroup (strongly continuous semigroup) $(\mathbb{T}(t))_{t \geq 0}$ on $X, B$ is unbounded linear control operators

Received by the editors in July 2012 - In revised form in June 2013.

Communicated by P. Godin.

2010 Mathematics Subject Classification : 34K30, 35R15, 39A14, 32A70, 93C25, 93C20.

Key words and phrases: Infinite-dimensional systems, semigroups, Favard spaces, unbounded linear (bilinear) control systems, admissibility, abstract linear (bilinear) control systems, boundary control systems. 
in the sense that it is a bounded linear operator from $X$ to a larger Banach space $V \supset X$, i.e. $B \in \mathcal{L}(X, V)$ (space of bounded linear operator from $X$ to $V$ ). The function $u \in L_{\text {loc }}^{p}\left(\mathbb{R}^{+}\right)$is a scalar control.

In order to give a meaning to the mild and strong solutions of (1.1) we have to suppose that the semigroup $(\mathbb{T}(t))_{t \geq 0}$ can be extended to a strongly continuous semigroup $\left(\mathbb{T}^{V}(t)\right)_{t \geq 0}$ on $V$ with generator $\left(A^{V}, \mathcal{D}\left(A^{V}\right)\right)$.

Thus, in the larger space $V$ the system (1.1) can be rewritten in the abstract form:

$$
\left\{\begin{array}{l}
\dot{x}(t)=A^{V} x(t)+u(t) B x(t), \quad t \geq 0 \\
x(0)=x_{0}
\end{array}\right.
$$

The initial state $x_{0} \in V$ is given. By a mild solution of (1.2) we mean an $X$-valued continuous function $x$ on $[0, T]$ satisfying (variation of parameters formula) :

$$
x(t)=\mathbb{T}^{V}(t) x_{0}+\int_{0}^{t} \mathbb{T}^{V}(t-s) u(s) B x(s) d s .
$$

All vector-valued integrals are Bochner integrals. Remark that the solution of (1.3) is always well-defined in $V$ for all $x_{0} \in V$ and $u \in L^{p}(0, T), T>0$. In general, due to the unboundedness aspect of the control operator $B$ this solution does not exist with values in the domain of $B$. Thus, to confront this difficulty, and inspired by the concept of admissibility developed in [29] for unbounded linear control systems, the author in [16] has introduced the following notion of admissibility: the operator $B$ is said to be $(p, q)$-admissible if the convolution $\Phi_{t}(u, x):=$ $\left(\mathbb{T}^{V} * u B x\right)(t) \in X$ for all $u \in L_{l o c}^{p}\left(\mathbb{R}^{+}\right), x \in L_{l o c}^{q}\left(\mathbb{R}^{+}, X\right)$ and $t \geq 0$ where $p$ and $q$ are conjugates. Unfortunately, it appears along the results obtained in [16] that this definition is too restrictive in the sense it does not concern the reflexive (e.g. Hilbert) state space $X$, the operator $B$ must be bounded makes this definition without interest. Further, unbounded bilinear systems in reflexive Banach spaces are not concerned, which makes hard to find examples of admissible operators enabling us to treat significant examples. In [2], the author extends this definition of admissibility for the operator $B$ by avoiding the constraint that $p$ and $q$ be conjugates, i.e. $\frac{1}{p}+\frac{1}{q}=1$ and by using the generalized Hölder inequality he deduces that $\Phi_{t}(u, x) \in X$ provided that

$$
0<\frac{1}{p}+\frac{1}{q} \leq 1
$$

thus the conjugacy constraint of $(p, q)$ dispensed with.

Further, it has been proved in [16] (see. [2] for conjugate case) that system (1.1) with a $(p, q)$-admissible control operator has a unique mild solution. Contrary to the author in [16], the concept of an abstract bilinear control systems has not been considered in [2]. Therefore, neither the representation theorem, nor the identification of such operators were studied in [2]. So, we are still longing for such a complete result regarding the $(p, q)$-admissibility of the unbounded control operators for infinite dimensional bilinear control systems, at least for such systems in Hilbert spaces and it is one the objectives of this paper. 
We proceed as follows: In Section 2 we recall the necessary background on extrapolation theory and Favard classes. In section 3 we review some results on the abstract linear and bilinear systems and some known (and unknown) of its properties. The main results of this paper are contained in Sections 4 and 5. In section 4 we give a relation between the space of $p$-admissible linear operators and some Favard class. This allows us the prove that the $p$-Weiss property is true for $p=1$. Secondly we pay particular attention to the analytic semigroups case. In [17] the authors have been interested in case $p=1$ for some boundary control systems and they have studied their reachability. In Section 5 we go back to the work of [17] and show that the considered sufficient (smoothness) condition is in fact necessary for the existence of the mild solutions. In section 6, an analogous result which links the space of $(p, q)$-admissible operators and the Favard class is the Proposition 24 first proved under the conjugacy of the reels $p$ and $q$ in [16]. Moreover, we pay particular attention to the analytic semigroups case in order to generalize a result in [2] on the $(p, q)$-admissibility of the fractional Laplacian $(-\Delta)^{\gamma}$ where $\Delta$ denotes the usual Dirichlet Laplacian operator for a system governed by the heat equation. Finally, in Section 7 we go back to such fractional diffusions system governed by the heat equation, and extend the results given in [2].

\section{Notions and preliminary results}

In this section we review some notions and results from the theory of extrapolation introduced in [24] and [21]. The details and proofs can be found, e.g., in [11].

\subsection{Extrapolation spaces and Favard classes}

Let $(X,\|\cdot\|)$ be a Banach space and $\mathbb{T}:=(\mathbb{T}(t))_{t \geq 0}$ be a $C_{0}$-semigroup of the bounded linear operators on $X$, with generator $(A, \mathcal{D}(A))$, and $\omega_{0}(\mathbb{T}):=$ $\inf \left\{\omega \in \mathbb{R} /\|\mathbb{T}(t)\|_{\mathcal{L}(X)} \leq M e^{\omega t}\right.$, for some $\left.M \geq 1\right\}$ its growth bound. The spaces $X_{1}$ and $X_{-1}$ are defined as follows: $X_{1}:=\left(\mathcal{D}(A),\|\cdot\|_{1}\right)$, where $\|x\|_{1}:=$ $\|(\lambda I-A) x\|, x \in \mathcal{D}(A)$ (for some $\lambda$ fixed in the resolvent set $\rho(A)$ of $A$ ), and $X_{-1}$ is the completion of $X$ with respect to the norm $\|x\|_{-1}:=\left\|(\lambda I-A)^{-1} x\right\|, x \in X$. These spaces are independent of the choice of $\lambda$ and are related by the following continuous and dense injections:

$$
X_{1} \stackrel{d}{\hookrightarrow} X \stackrel{d}{\hookrightarrow} X_{-1}
$$

The Banach space $X_{-1}$ is called the extrapolation space of $X$ with respect to $A$ (or $\mathbb{T}$ ). If there is confusion on $A$ we use the notations $X_{1}^{A}$ and $X_{-1}^{A}$. The semigroup $(\mathbb{T}(t))_{t \geq 0}$ can be restricted to a $C_{0}$-semigroup $\mathbb{T}_{1}$ on $X_{1}$, with generator denoted by $\left(A_{1}, \mathcal{D}\left(A_{1}\right)\right)$, and can be extended to a $C_{0}$-semigroup $\left(\mathbb{T}_{-1}(t)\right)_{t \geq 0}$ on $X_{-1}$, with generator denoted by $\left(A_{-1}, \mathcal{D}\left(A_{-1}\right)\right)$. The resolvent operator $R(\lambda, A):=(\lambda I-A)^{-1}$ and its extension $R\left(\lambda, A_{-1}\right)$ to $X_{-1}$ are isomorphisms from $X$ to $X_{1}$ and from $X_{-1}$ to $X$ respectively. In particular, $\mathcal{D}\left(A_{-1}\right)=X$ and the 
norm of $X$ is equivalent to the graph norm of $A_{-1}$.

We define the Favard class of order $\alpha$; $F_{A}^{\alpha}$ with $0<\alpha \leq 1$ associated to $A$ (or $\left.(\mathbb{T}(t))_{t \geq 0}\right)$ by

$$
F_{A}^{\alpha}:=\left\{x \in X / \lim _{t \searrow 0} \sup \frac{1}{t^{\alpha}}\|\mathbb{T}(t) x-x\|<+\infty\right\}
$$

([7], [23]) and similarly the Favard class $F_{A_{-1}}^{\alpha}$ of $\left(\mathbb{T}_{-1}(t)\right)_{t \geq 0}$. An equivalent definition of Favard class of order $\alpha$ used here is the following

$$
F_{A}^{\alpha}=\left\{x \in X / \sup _{0<t \leq 1} \frac{1}{t^{\alpha}}\left\|e^{-\omega t} \mathbb{T}(t) x-x\right\|<+\infty\right\},
$$

with $\omega>\omega_{0}(\mathbb{T})$. It is a Banach space equipped with norm

$$
\|x\|_{F_{A}^{\alpha}}:=\|x\|+\sup _{t>0} \frac{1}{t^{\alpha}}\left\|e^{-\omega t} \mathbb{T}(t) x-x\right\|,
$$

and similarly $\|x\|_{F_{A_{-1}}^{\alpha}}$ on $F_{A_{-1}}^{\alpha}$. We note that $\sup _{\lambda>\omega}\left\|\lambda^{\alpha} A R(\lambda, A) x\right\|$ is an equivalent norm on $F_{A}^{\alpha}$ (independent of the choice of $\omega$ ), invariant under $(\mathbb{T}(t))_{t \geq 0}$ and $X_{1} \hookrightarrow F_{A}^{\alpha} \hookrightarrow X$. Obviously, $\lambda I-A$ maps $F_{A}^{\alpha}$ isometrically onto $F_{A_{-1}}^{\alpha}$. If $X$ is reflexive Banach space then $F_{A}^{1}:=F_{A}=\mathcal{D}(A)$ (see. e.g. [11]). Actually, the Favard class $F_{A}^{\alpha}$ can be defined for $\alpha$ in the range $(0, \infty)$. We will restrict our considerations to the case $\alpha \in(0,1]$.

For analytic semigroups these spaces are characterized as follows (see. e.g. [11]):

Proposition 1. Assume that A generates an analytic semigroup $(\mathbb{T}(t))_{t \geq 0}$ on a Banach space $X$ with $\omega_{0}(\mathbb{T})<0$. If $\alpha \in(0,1]$, on has

$$
F_{A}^{\alpha}=\left\{x \in X / \sup _{t>0}\left\|t^{1-\alpha} A \mathbb{T}(t) x\right\|<+\infty\right\}
$$

and the Favard norm $\|\cdot\|_{F_{A}^{\alpha}}$ is equivalent to the norm

$$
|x|_{F_{A}^{\alpha}}:=\sup _{t>0}\left\|t^{1-\alpha} A \mathbb{T}(t) x\right\| .
$$

Let $E$ be Banach space, we will use the following notations: $\mathbb{R}^{+}=[0,+\infty[$; $E^{p}=L^{p}\left(\mathbb{R}^{+}, E\right) ; E_{l o c}^{p}=L_{\text {loc }}^{p}\left(\mathbb{R}^{+}, E\right) ; E_{t}^{p}=L^{p}(0, t ; E)$ with $p \in[1,+\infty] . \mathbb{C}_{\omega}=$ $\{s \in \mathbb{C} / \operatorname{Re}(s)>\omega\}$. For a fixed $p \in[1,+\infty]$ we denote by $p^{\prime}$ the dual exponent given by $1 / p+1 / p^{\prime}=1$, and we say that $p$ and $p^{\prime}$ are conjugates. Throughout the paper, the norm of any other Banach space, say $E$, will be specified by $\|\cdot\|_{E}$. In this work, we use the same letter $K$ to denote different constants. The value of $K$ can change from one expression to another.

We will need the following crucial lemma which is due to ([9],Theorem.9). A similar version can be found in ([22], Prop. 3.3) and ([23], Lemma. 4.3.9). 
Lemma 2. Let $(\mathbb{T}(t))_{t \geq 0}$ be a $C_{0}$-semigroup of bounded linear operators on $X$, with generator $(A, \mathcal{D}(A))$. For all $f \in L_{\text {loc }}^{1}\left(0,+\infty ; F_{A}\right)$ and $t \geq 0$, we set

$$
(\mathbb{T} * f)(t):=\int_{0}^{t} \mathbb{T}(t-s) f(s) d s .
$$

Then one has

1. $(\mathbb{T} * f)(t) \in \mathcal{D}(A)$,

2. For $\omega>\omega_{0}(\mathbb{T})$, there is a constant $K$, independent of $(t, f)$, such that

$$
\|(\mathbb{T} * f)(t)\|_{\mathcal{D}(A)} \leq K e^{(|\omega| t)}\|f\|_{L^{1}\left(0, t ; F_{A}\right)},
$$

where $\|\cdot\|_{\mathcal{D}(A)}$ denotes the graph norm of $A$, i.e. $\|x\|_{\mathcal{D}(A)}=\|x\|+\|A x\|, x \in \mathcal{D}(A)$.

Definition 3. Let $\tau>0$ and $f, g$ be two functions of $E_{l o c}^{p}(1 \leq p \leq+\infty)$, where $E$ is a Banach space. The $\tau$-concatenation of $f$ and $g$ is the function $f \underset{\tau}{\gamma} g \in E_{l o c}^{p}$ given by

$$
(f \underset{\tau}{f} g)(t)= \begin{cases}f(t) & ; \quad 0 \leq t<\tau \\ g(t-\tau) & ; t \geq \tau\end{cases}
$$

\section{Framework on Abstract linear and bilinear control systems}

In this section, we only state some necessary concepts (in particular admissibility and representations theorems) for abstract control linear and bilinear systems. The notations and terminologies used herein agree almost completely with that of [25], [29] and [16], [3] and [2] respectively. But for the readers' convenience, we repeat some of them.

\subsection{Abstract linear control systems}

Definition 4. An abstract linear control system (ALCS for short) for $X, U^{p}$ with $p \in[1,+\infty]$ is a pair $(\mathbb{T}, \boldsymbol{\Phi})$, where $\mathbb{T}:=(\mathbb{T}(t))_{t \geq 0}$ be a $C_{0}$-semigroup of bounded linear operators on $X$ and $\boldsymbol{\Phi}=\left(\boldsymbol{\Phi}_{t}\right)_{t \geq 0}$ is a family of bounded linear operators from $U^{p}$ to $X$ (i.e., $\left.\boldsymbol{\Phi}_{t} \in \mathcal{L}\left(U^{p}, X\right)\right)$ such that:

$$
\boldsymbol{\Phi}_{\tau+t}(\underset{\tau}{u \diamond v})=\mathbb{T}(t) \boldsymbol{\Phi}_{\tau} u+\boldsymbol{\Phi}_{t} v
$$

for any $u$ and $v$ in $U^{p}$ and $t, \tau \geq 0$.

The functional equation (3.1) is called the composition property.

Definition 5. Let $(\mathbb{T}(t))_{t \geq 0}$ be a $C_{0}$-semigroup of bounded linear operators on $X$, with generator $(A, \mathcal{D}(A))$, and let $V$ be a Banach space such that:

1. $X \stackrel{d}{\hookrightarrow} V$ (i.e. continuous and dense injection). 
2. $\mathbb{T}(t)$ has an extension $\mathbb{T}^{V}(t)$ on $V$ which forms a $C_{0}$-semigroup on $V$.

We say that $B \in \mathcal{L}(U, V)$ is an $p$-admissible $(p \in[1,+\infty])$ control operator for $(\mathbb{T}(t))_{t \geq 0}($ or $A)$, if

$$
\int_{0}^{t} \mathbb{T}^{V}(t-s) B u(s) d s \in X
$$

for all $u$ in $U_{l o c}^{p}$ and $t \geq 0$. We note that the extension $C_{0}$-semigroup $\left(\mathbb{T}^{V}(t)\right)_{t \geq 0}$, if it exists it is unique, with its generator denoted by $\left(A^{V}, \mathcal{D}\left(A^{V}\right)\right) . B \in \mathcal{L}(U, V)$ is said to be a weakly $p$-admissible control operator for $(\mathbb{T}(t))_{t \geq 0}$; if for every $v \in U$, the vector $b_{v}:=B v$ is a $p$-admissible for $(\mathbb{T}(t))_{t \geq 0}$. i.e., (3.2) holds for all $u$ of the form $u(\sigma)=v w(\sigma)$, where $v \in U$ and $w \in L^{p}(0 ; t ; \mathbb{C})$.

The closed graph theorem shows that if $B$ is $p$-admissible control operator for $(\mathbb{T}(t))_{t \geq 0}$ then, for some ( and hence all) $t \geq 0$ there is $K:=K_{t} \geq 0$ such that

$$
\left\|\int_{0}^{t} \mathbb{T}^{V}(t-s) B u(s) d s\right\| \leq K\|u\|_{U_{t}^{p}}
$$

for all $u \in U_{l o c}^{p}$ with $p \in[1,+\infty]$. And the operator which belongs to $\mathcal{L}\left(U^{p}, V\right)$, defined by

$$
\Phi_{t}^{V}(u):=\int_{0}^{t} \mathbb{T}^{V}(t-s) B u(s) d s
$$

is also bounded from $U^{p}$ to $X$. It is well-known that the couple $\left(\mathbb{T}, \boldsymbol{\Phi}^{\mathbf{V}}\right)$ is an ALCS for $X, U^{p}$.

Definition 6. Let $(\mathbb{T}(t))_{t \geq 0}, X$ and $V$ defined as in Definition 5 . We denote by $\mathcal{A}_{p}(U, X, V, \mathbb{T})$ with $p \in[1,+\infty]$ the space of all $p$-admissible control operators for $(\mathbb{T}(t))_{t \geq 0}$. We endow this space with the norm

$$
\|B\|_{p}^{V, t}:=\sup _{\|u\|_{U_{t}^{p}} \leq 1}\left\{\left\|\int_{0}^{t} \mathbb{T}^{V}(t-s) B u(s) d s\right\|\right\}, t>0 .
$$

The following direct result is needed for a part of the proof of Proposition 15.

Claim. In the conditions of Definition 5 we have $\|B\|_{p}^{V, t}=\left\|\Phi_{t}^{V}\right\|_{\mathcal{L}(\mathcal{U} \sqrt{ }, \mathcal{X})}$, where $\Phi^{V}$ is given by (3.3).

Proof. By using the property of causality verified by the integral operator $\Phi_{t}^{V}$ we obtain

$$
\|B\|_{p}^{V, t}=\sup _{\|u\|_{u_{t}^{p} \leq 1}}\left\{\left\|\boldsymbol{\Phi}_{t}^{V}(\underset{t}{u \gg 0})\right\|\right\}
$$

Since $\|\underset{t}{u \diamond 0}\|_{\mathcal{U}^{p}}=\|u\|_{U_{t}^{p}}$ we obtain $\|B\|_{p}^{V, t}=\left\|\Phi_{t}^{V}\right\|_{\mathcal{L}(\mathcal{U} \sqrt{ }, \mathcal{X})}$.

We denote $\mathcal{A}_{p}(U, X, \mathbb{T}):=\mathcal{A}_{p}\left(U, X, X_{-1}, \mathbb{T}\right)$ and the set $\mathfrak{a}_{p}(X, \mathbb{T}):=$ $\mathcal{A}_{p}\left(\mathbb{C}, X, X_{-1}, \mathbb{T}\right)$ denotes the space of $p$-admissible input elements for $(\mathbb{T}(t))_{t \geq 0}$. 
The following representation theorem ensures the existence and the uniqueness of a $p$-admissible control operator associated with an ALCS. For the proof we refer the reader to [29].

Theorem 7. Let $(\mathbb{T}(t))_{t \geq 0}$ be a $C_{0}$-semigroup on the Banach space $X$ generated by $(A, \mathcal{D}(A))$ and let $(\mathbb{T}, \boldsymbol{\Phi})$ be an $A L C S$. for $X, U^{p}$ with $p \in[1,+\infty[$. Then, there is a unique operator $B \in \mathcal{L}\left(U, X_{-1}\right)$ such that

$$
\boldsymbol{\Phi}_{t} u=\int_{0}^{t} \mathbb{T}_{-1}(t-s) B u(s) d s,
$$

for any $u \in U^{p}$ and $t \geq 0$.

Thus if $B \in \mathcal{L}(U, V)$ be a $p$-admissible control operator, then by Theorem 7 there exists a unique operator $B_{0} \in \mathcal{L}\left(U, X_{-1}\right)$ such that

$$
\boldsymbol{\Phi}_{t}^{V} u=\int_{0}^{t} \mathbb{T}_{-1}(t-s) B_{0} u(s) d s,
$$

for any $u \in U^{p}$ and $t \geq 0$.

Remark 8. In general, $B_{0}$ is not equal to $B$ (see e.g. [29], [8]). However, if $A$ has a continuous extension to an operator in $\mathcal{L}(X, V)$ (e.g. $\left.X \hookrightarrow \mathcal{D}\left(A^{V}\right)\right)$ then $B_{0}=B$.

For the next, recall that in Proposition 2.3 [30] (resp. in the Proposition 4.2.9 [27]) it has been proved that for $p=2$ (resp. $p \in[1,+\infty]$ ) the following $p$ resolvent condition

$$
\left\|\left(s I-A_{-1}\right)^{-1} B\right\|_{\mathcal{L}(\mathcal{U}, \mathcal{X})} \leq \frac{K}{\operatorname{Re}(\mathrm{s})^{1 / \mathrm{p}}}, s \in \mathbb{C}_{\omega} .
$$

is necessary for the $p$-admissibility of the operator $B$.

This leads to the following definition which is inspired by the Weiss conjectures.

Definition 9. Let $A$ generate a bounded $C_{0}$-semigroup on a Banach $X$ and $U$ is an other Banach space and $p \in[1,+\infty]$. We say that $A$ satisfies the $p$-Weiss property if for any $B \in \mathcal{L}\left(U ; X_{-1}\right)$ the following statements are equivalent:

1. $B$ is a $p$-admissible control operator for $(\mathbb{T}(t))_{t \geq 0}$.

2. $B$ satisfies the $p$-resolvent condition (3.5).

In the literature there are many results concerning the validity or invalidity of the 2-Weiss property on Hilbert spaces for some classes of $C_{0}$-semigroups (see, e.g. [18],[6]). As far as the analytic semigroups are concerned, it has been shown in [18] and in [4] for a short proof (resp. in [13], [4], [5]) that 2-Weiss (resp. $p$-Weiss, $p \in[1,+\infty]$ ) property holds if and only if $(-A)^{1 / 2}$ is 2-admissible (resp. $(-A)^{1 / p}$ is $p$-admissible) and generally the 2-Weiss property no longer holds even for compact semigroups (see. [14]). 
Remark 10. The following simple observation will be helpful for the rest: The fact that the notion of $p$-admissibility is invariant under scalings $e^{-\alpha \cdot} \mathbb{T}$, to investigate the $p$-admissibility we may assume that $\mathbb{T}$ is exponentially stable. Thus for $B \in \mathcal{L}\left(U, X_{-1}\right)$ and for real $s$ large enough and by definition of the extrapolation space $X_{-1}$ (with $\lambda=0$ ) we obtain

$$
\left\|s^{1 / p} A_{-1}\left(s I-A_{-1}\right)^{-1} B u\right\|_{-1}=\left\|s^{1 / p}\left(s I-A_{-1}\right)^{-1} B u\right\| \quad p \geq 1 .
$$

Notice that the above condition can be reformulated by saying that if $B$ is $p$ admissible then $B u \in F_{A_{-1}}^{1 / p}$ for all $u \in U$ accordingly to (3.5). Finally, the closed graph theorem implies that a new necessary condition of $p$-admissibility of $B$ for $(\mathbb{T}(t))_{t \geq 0}$ is

$$
\operatorname{Range}(B) \subset F_{A_{-1}}^{1 / p}
$$

and in an implicit setting it was observed in Remark $3.3[29]$ that $\mathfrak{a}_{2}(X, \mathbb{T}) \subset F_{A_{-1}}^{\frac{1}{2}}$.

So, for spaces $X$ and $V$ as in Definition 5 we can ask: "under what conditions on the underlying semigroup $(\mathbb{T}(t))_{t \geq 0}$ the equality :

$$
\mathcal{L}\left(U, F_{A^{V}}^{1 / p}\right)=\mathcal{A}_{p}(U, X, V, \mathbb{T}),
$$

takes place"?

For analytic semigroups it is easy to see that the $p$-resolvent condition is true if and only if it is for real $s$ only. Thus, with $V=X_{-1}$ a necessary and sufficient condition for a semigroup to satisfy the $p$-Weiss property is that $\mathcal{A}_{p}(U, X, \mathbb{T})=$ $\mathcal{L}\left(U, F_{A_{-1}}^{1 / p}\right)$ accordingly to identity (3.6).

For a normal (resp. bounded) and analytic semigroup $(\mathbb{T}(t))_{t \geq 0}$ on a Hilbert (resp. on Banach) space $X$, Remark 3.3 in [29] (resp. Theorem 4.1 [18] and Theorem 1.8 [13]) implicitly contains an affirmative answer for $p=2$ and for $p=1$ respectively. In particular for an analytic semigroup on $X=l^{r}$, the Theorem 1.1 [28] implicitly contains an affirmative answer with $V=X_{-1}$ for all $p$ with $r \geq p^{\prime} \geq 2$. For all semigroups $(\mathbb{T}(t))_{t \geq 0}$ on a reflexive Banach space $X$ an affirmative answer for $p=1$ was given in the Theorem 4.8 [29] since in this case $F_{A_{-1}}=X$ as cited above. Below we show that Theorem 4.8 [29] takes place for all Banach space.

Unlike identity (3.8), in the next section and in spite of the invalidity of (3.6) we will show that the condition (3.7) remains true for all pairs $(X, V)$ as in Definition 5 with an extra assumption.

\subsection{Abstract Bilinear Control Systems}

In this subsection we recall the definition of an abstract bilinear control systems. It is stated in [16] for the conjugated $p$ and $q$. But for the non-conjugated $p$ and $q$ satisfying (1.4) the author in [2] deals only with the definition of $(p, q)$-admissible bilinear control operators. 
In the sequel, $p$ and $q$ are real numbers in $[1,+\infty]$ satisfying (1.4).

Definition 11. An abstract bilinear control system (ABCS for short) for $\mathbb{C}^{p} \times X^{q}$ is a pair $(\mathbb{T}, \Psi)$, where $\mathbb{T}:=(\mathbb{T}(t))_{t \geq 0}$ be a $C_{0}$-semigroup of bounded linear operators on $X$ and $\Psi=\left(\Psi_{t}\right)_{t>0}$ is a family of bounded bilinear operators from $\mathbb{C}^{p} \times X^{q}$ to $X$ (i.e., $\left.\Psi_{t} \in \mathcal{B} \mathcal{L}\left(\mathbb{C}^{p} \times X^{q}, X\right)\right)$ such that

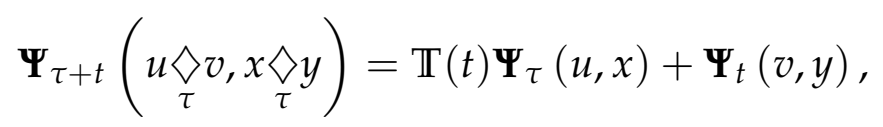

for any $u, v \in \mathbb{C}^{p}, x, y \in X^{q}$ and $t, \tau \geq 0$.

The functional equation (3.9) is called the "composition property".

The following definition was introduced in [16] for conjugates $p$ and $q$ and in [2] for all $(p, q)$ satisfying (1.4).

Definition 12. Let $(\mathbb{T}(t))_{t \geq 0}, X$ and $V$ defined as in Definition 5 , we say that $B \in \mathcal{L}(X, V)$ is an $(p, q)$-admissible operator control for $(\mathbb{T}(t))_{t \geq 0}($ or $A)$, if

$$
\int_{0}^{t} \mathbb{T}^{V}(t-s) u(s) B x(s) d s \in X
$$

for all $u, x$ in $\mathbb{C}_{l o c}^{p} \times X_{l o c}^{q}$ and $t \geq 0$.

We note that a $(p, q)$-admissible operator $B$ is also a $q$-admissible control operator for $(\mathbb{T}(t))_{t \geq 0}$ (this can be seen by considering the input $u=1$ ). If $B$ is a $(p, q)$ admissible control operator for $(\mathbb{T}(t))_{t \geq 0}$, then for all $t \geq 0$ there is $K:=K_{t} \geq 0$ such that

$$
\left\|\int_{0}^{t} \mathbb{T}^{V}(t-s) u(s) B x(s) d s\right\|_{X} \leq K\|u\|_{\mathbb{C}_{t}^{p}}\|x\|_{X_{t}^{q}},
$$

for all $(u, x) \mathbb{C}_{l o c}^{p} \times X_{l o c}^{q}$.

As in the linear case, we have the following representation theorem which has been proved in [16] where $p$ and $q$ are conjugates. The proof can be obtained similarly for non conjugates $p$ and $q$ and it is omitted.

Theorem 13. Let $(\mathbb{T}(t))_{t \geq 0}$ be a $C_{0}$-semigroup on the Banach space $X$ generated by $(A, \mathcal{D}(A))$. Let $(\mathbb{T}, \Psi)$ be an $A B C S$ for $\mathbb{C}^{p} \times X^{q}$ with $\left.p, q \in\right] 1,+\infty[$ satisfying (1.4). Then, there is a unique operator $B \in \mathcal{L}\left(X, X_{-1}\right)$ such that:

$$
\Psi_{t}(u, x)=\int_{0}^{t} \mathbb{T}_{-1}(t-s) u(s) B x(s) d s,
$$

for any $(u, x)$ in $\mathbb{C}^{p} \times X^{q}$ and $t \geq 0$.

Remark 14. Let $B \in \mathcal{L}(X, V)$ and be $(p, q)$-admissible control operator for $(\mathbb{T}(t))_{t \geq 0}$ we have the following statements: 
(i) If we denote

$$
\Psi_{t}^{V}(u, x)=\int_{0}^{t} \mathbb{T}^{V}(t-s) u(s) B x(s) d s
$$

with $(u, x)$ in $\mathbb{C}^{p} \times X^{q}$ then the couple $\left(\mathbb{T}, \Psi^{V}\right)$ is an ABCS for $\mathbb{C}^{p} \times X^{q}$. By representation Theorem 13 there is a unique operator $B_{0} \in \mathcal{L}\left(X, X_{-1}\right)$ such that

$$
\Psi_{t}^{V}(u, x)=\int_{0}^{t} \mathbb{T}_{-1}(t-s) u(s) B_{0} x(s) d s,
$$

for any $(u, x)$ in $\mathbb{C}^{p} \times X^{q}$ and $t \geq 0$.

(ii) When $A^{V} \in \mathcal{L}(X, V)$, we deduce that $B_{0}=B$ which is due to the fact that $B$ is a $q$-admissible control operator for $(\mathbb{T}(t))_{t \geq 0}$, (see. Remark 8).

\section{Admissible Linear Control Operators and the Favard spaces}

After recalling the definitions of ALCS and some related theorems, this section states the main results giving a relationship between the space of the admissible control operators and the Favard spaces.

Proposition 15. Let $U$ Banach space and let $X, V$ and $(\mathbb{T}(t))_{t \geq 0}$ as defined in Definition 5 and $p \in\left[1,+\infty\right.$. If there is an isomorphism between $\mathcal{D}\left(A^{V}\right)$ and $X$ then:

$$
\mathcal{L}\left(U, F_{A^{V}}\right) \hookrightarrow \mathcal{A}_{p}(U, X, V, \mathbb{T}) \hookrightarrow \mathcal{L}\left(U, F_{A^{V}}^{\frac{1}{p}}\right),
$$

where $F_{A^{V}}$ denotes the Favard space associated with $A^{V}$.

Remark 16. For Banach spaces $X$ and $V$ as in Proposition 15 we don't necessary have a similar identity to (3.6) but we always have $\mathcal{A}_{p}(U, X, V, \mathbb{T}) \subset \mathcal{L}\left(U, F_{A_{-1}}^{\frac{1}{p}}\right)$. In fact, let $B \in \mathcal{A}_{p}(U, X, V, \mathbb{T})$ then by virtue of Remarks 8 and 10 we obtain $B \in \mathcal{A}_{p}(U, X, \mathbb{T}) \subset \mathcal{L}\left(U, F_{A_{-1}}^{\frac{1}{p}}\right)$.

Proof. (Proof of Proposition 15). Let $B \in \mathcal{L}\left(U, F_{A^{V}}\right)$ and $u \in U_{l o c}^{p} \subseteq U_{l o c}^{1}$ then $B u(\cdot) \in L_{l o c}^{1}\left(\mathbb{R}^{+}, F_{A^{V}}\right)$ and by Lemma 2 (i) we have

$$
\int_{0}^{t} \mathbb{T}^{V}(t-s) B u(s) d s \in \mathcal{D}\left(A^{V}\right),
$$

for all $t \geq 0$, since $\mathcal{D}\left(A^{V}\right) \simeq X$ this implies $B \in \mathcal{A}_{p}(U, X, V, \mathbb{T})$ and by Lemma 2 (ii) there is $K:=K_{t}>0$ such that:

$$
\left\|\int_{0}^{t} \mathbb{T}^{V}(t-s) B u(s) d s\right\| \leq K\|B\|_{\mathcal{L}\left(U, F_{A} V\right.}\|u\|_{L^{1}(0, t ; U)}
$$

and since $U_{l o c}^{p} \subseteq U_{l o c}^{1}$ there is $K:=K_{t}>0$ such that

$$
\left\|\int_{0}^{t} \mathbb{T}^{V}(t-s) B u(s) d s\right\| \leq K\|B\|_{\mathcal{L}\left(U, F_{A} V\right)}\|u\|_{L^{p}(0, t ; U)}
$$


which implies that

$$
\left.\|B\|_{p}^{V, t} \leq K\|B\|_{\mathcal{L}\left(U, F_{A} V\right.}\right)
$$

On an other hand, let $B \in \mathcal{A}_{p}(U, X, V, \mathbb{T})$. Since $F_{A^{V}}^{1 / p} \hookrightarrow V$, it is enough, by the closed graph theorem, to show that $\operatorname{Range}(B) \subset F_{A^{V}}^{1 / p}$. So, let $u \in U$ and $t>0$. As $X \hookrightarrow D\left(A^{V}\right)$, one can write $\left(A_{\omega}^{V}:=A^{V}-\omega I\right)$

$$
\begin{aligned}
\left\|e^{-\omega t} \mathbb{T}^{V}(t) B u-B u\right\|_{V} & =\left\|A_{\omega}^{V} \int_{0}^{t} e^{-\omega s} \mathbb{T}^{V}(s) B u d s\right\|_{V} \\
& \leq K\left\|\int_{0}^{t} e^{-\omega s} \mathbb{T}^{V}(s) B u d s\right\| \\
& \leq K t^{1 / p}\left\|\Phi_{t}^{V}\right\|_{p}\|u\| .
\end{aligned}
$$

The fact that $\left\|\Phi_{t}^{V}\right\|_{\mathcal{L}(\mathcal{U}, \mathcal{X})}$ (well-known result [29]) is monotonically increasing in $t$ and using the above Claim, it follows that

$$
\sup _{t \in] 0,1]} \frac{1}{t^{1 / p}}\left\|e^{-\omega t} \mathbb{T}^{V}(t) B u-B u\right\|_{V} \leq K\|B\|_{p}^{V, 1}\|u\|
$$

Thus, $B u \in F_{A^{V}}^{1 / p}$ and we have

$$
\|B\|_{\mathcal{L}\left(U, F_{A V}^{1 / p}\right)} \leq K\|B\|_{p}^{V, 1}
$$

We are now in a position to state the main result of this subsection which generalizes the result recalled at the end of the subsection 3.1 concerning $p=1$. More than that, it is of great use in the paper [6].

Corollary 17. In the conditions of Proposition 15 we have

$$
\mathcal{A}_{1}(U, X, V, \mathbb{T})=\mathcal{L}\left(U, F_{A^{V}}\right) .
$$

In particular $\mathcal{A}_{1}(U, X, \mathbb{T})=\mathcal{L}\left(U, F_{A_{-1}}\right)$, thus the $p$-Weiss property is true for $p=1$.

Corollary 18. Let $X, V$ and $(\mathbb{T}(t))_{t \geq 0}$ as in Proposition 15. Then we have $F_{A^{V}} \hookrightarrow F_{A_{-1}}$.

Proof. Let $b \in F_{A^{V}}$. Appealing to Corollary 17 we obtain $b \in \mathfrak{a}_{1}(X, V, \mathbb{T})$ and using Remark 8 we obtain $b \in \mathfrak{a}_{1}(X, \mathbb{T})=F_{A_{-1}}$.

In the conditions of Corollary 18 a similar result for the pair $\left(F_{A_{-1}}^{1 / p} F_{A^{V}}^{1 / p}\right)$ for $p \in] 1,+\infty\left[\right.$ cannot be announced but we infer that we only have $\mathfrak{a}_{p}(X, V, \mathbb{T}) \subset$ $F_{A_{-1}}^{1 / p} \cap F_{A^{V}}^{1 / p}$.

We now take a look at the situation where $A$ generates an analytic semigroup on $X$ and $p>1$.

Proposition 19. Let $A$ generate a bounded analytic semigroup $(\mathbb{T}(t))_{t \geq 0}$ on a Banach space $X$. Then for all $\beta \in\left(\frac{1}{p}, 1\right]$ we have:

$$
\mathcal{L}\left(U, F_{A_{-1}}^{\beta}\right) \hookrightarrow \mathcal{A}_{p}(U, X, \mathbb{T}) \hookrightarrow \mathcal{L}\left(U, F_{A_{-1}}^{1 / p}\right)
$$


Proof. Let $B \in \mathcal{L}\left(U, F_{A_{-1}}^{\beta}\right)$ and $u \in U_{l o c}^{p}$ then

$$
\begin{aligned}
\| \int_{0}^{t} \mathbb{T}_{-1}(t & -s) B u(s) d s \| \\
& =\left\|\int_{0}^{t}\left(-A_{-1}\right)^{-1}(t-s)^{1-\beta}\left(-A_{-1}\right)(t-s)^{\beta-1} \mathbb{T}_{-1}(t-s) B u(s) d s\right\| \\
& \leq \int_{0}^{t}\left\|\left(-A_{-1}\right)^{-1}(t-s)^{1-\beta}\left(-A_{-1}\right)(t-s)^{\beta-1} \mathbb{T}_{-1}(t-s) B u(s)\right\| d s \\
& =\int_{0}^{t}\left\|(t-s)^{1-\beta}\left(-A_{-1}\right)(t-s)^{\beta-1} \mathbb{T}_{-1}(t-s) B u(s)\right\|_{-1} d s .
\end{aligned}
$$

There is no loss of generality in assuming that $(\mathbb{T}(t))_{t \geq 0}$ is a bounded and so is $\left(\mathbb{T}_{-1}(t)\right)_{t \geq 0}$. Since Range $(B) \subset F_{A_{-1}}^{\beta}$ by virtue of Proposition 1 , we have

$$
\left\|(t-s)^{1-\beta}\left(-A_{-1}\right) \mathbb{T}_{-1}(t-s) B u(s)\right\|_{-1} \leq\|B\|_{\mathcal{L}\left(U, F_{A_{-1}}^{\beta}\right)}\|u(s)\| .
$$

Thus

$$
\left\|\int_{0}^{t} \mathbb{T}_{-1}(t-s) B u(s) d s\right\| \leq\|B\|_{\mathcal{L}\left(U, F_{A_{-1}}^{\beta}\right)} \int_{0}^{t}(t-s)^{\beta-1}\|u(s)\| d s,
$$

and it is easy to verify by Hölder inequality and taking into account the condition on $\beta$ that the scalar function $s \mapsto(t-s)^{\beta-1}\|u(s)\|$ is $L_{l o c}^{1}\left(\mathbb{R}^{+}\right)$then $B$ is $p$-admissible for $\mathbb{T}(t)$ and there is $K:=K_{t}>0$ such that

$$
\|B\|_{p}^{X_{-1}, t} \leq K\|B\|_{\mathcal{L}\left(U, F_{A_{-1}}^{\beta}\right)} .
$$

We mention that in the above proposition the embedding result on the space $\mathcal{A}_{p}(U, X, \mathbb{T})$ is optimal in the sense that the limit case $\beta=1 / p$ is also true if and only if the semigroup $\mathbb{T}(t)$ satisfies the $p$-Weiss property or equivalently the operator $\left(-A^{*}\right)^{1 / q}$ is $q$-admissible if $X$ is a reflexive Banach space (see. [5]).

\section{Application to Boundary Control Systems}

\section{Consider}

1. three Banach spaces $X, \partial X$ and $U$, called the state space, boundary space and control space, respectively.

2. a closed, densely defined system operator $A_{m}: \mathcal{D}\left(A_{m}\right) \subseteq X \rightarrow X$.

3. a boundary operator $Q \in \mathcal{L}\left(\mathcal{D}\left(A_{m}\right), \partial X\right)$.

4. a control operator $B \in \mathcal{L}(U, \partial X)$.

For these operators and spaces and a control function $u \in U_{l o c}^{1}$ and $x_{0} \in X$, we consider the abstract Cauchy problem with a boundary control

$$
\begin{cases}\dot{x}(t)=A_{m} x(t), & t \geq 0 \\ Q x(t)=B u(t), & t \geq 0 \\ x(0)=x_{0} & \end{cases}
$$


In order to investigate (5.1) we make the following assumption ensuring in particular that the uncontrolled abstract Cauchy problem, i.e., (5.1) with $B=0$, is well-posed.

In the spirit of Greiner's approach [12] (see. also [25]) and based on some results in [17] we assume that

1. the operator $A:=A_{m \mid \operatorname{ker} Q}$ defined as the restriction of $A_{m}$ to $\operatorname{ker} Q$ generates a strongly continuous semigroup $(\mathbb{T}(t))_{t \geq 0}$ on a non reflexive $X$.

2. the boundary operator $Q: \mathcal{D}\left(A_{m}\right) \rightarrow \partial X$ is onto.

Under these assumptions the following properties have been shown by [[12]; Lemmas 1.2, 1.3].

1. For each $\lambda \in \rho(A), \mathcal{D}\left(A_{m}\right)=\mathcal{D}(A) \oplus \operatorname{ker}\left(\lambda-A_{m}\right)$;

2. $Q_{\mid \operatorname{ker}\left(\lambda-A_{m}\right)}$ is invertible and the operator $Q_{\lambda}:=\left(Q_{\mid \operatorname{ker}\left(\lambda-A_{m}\right)}\right)^{-1}: \partial X \rightarrow$ $\operatorname{ker}\left(\lambda-A_{m}\right) \subseteq X$ is bounded;

3. $P_{\lambda}:=Q_{\lambda} Q \in \mathcal{L}\left(\mathcal{D}\left(A_{m}\right)\right)$ is a projection onto $\operatorname{ker}\left(\lambda-A_{m}\right)$ along $\mathcal{D}(A)$;

4. $R(\mu, A) Q_{\lambda}=\frac{1}{\lambda-\mu}\left(Q_{\mu}-Q_{\lambda}\right)=R(\lambda, A) Q_{\mu}$ for all $\lambda, \mu \in \rho(A), \lambda \neq \mu$.

The operator $Q_{\lambda}$ is called the Dirichlet operator for $\lambda \in \rho(A)$.

By defining the operators $B_{\lambda}:=Q_{\lambda} B \in \mathcal{L}\left(U, \operatorname{ker}\left(\lambda-A_{m}\right)\right)$ which are essential to obtain an explicit representation of the boundary control system (5.1) as follows:

$$
\left\{\begin{array}{l}
\dot{x}(t)=A\left(x(t)-B_{\lambda} u(t)\right)+\lambda B_{\lambda} u(t), \quad t \geq 0, \\
x(0)=x_{0} .
\end{array}\right.
$$

In the bigger space $X_{-1}$ the above system can be rewritten in the form:

$$
\left\{\begin{array}{l}
\dot{x}(t)=A_{-1} x(t)+\left(\lambda-A_{-1}\right) B_{\lambda} u(t), \quad t \geq 0 \\
x(0)=x_{0} .
\end{array} .\right.
$$

The initial state $x_{0} \in X$ is given. By a mild solution of (5.3) we mean a continuous function $x(t)$ on $[0, T]$ satisfying (variation of parameters formula) :

$$
\begin{aligned}
x(t) & :=\mathbb{T}(t) x_{0}+\int_{0}^{t} \mathbb{T}_{-1}(t-s)\left(\lambda-A_{-1}\right) B_{\lambda} u(s) d s \\
& =\mathbb{T}(t) x_{0}+\left(\lambda-A_{-1}\right) \int_{0}^{t} \mathbb{T}(t-s) B_{\lambda} u(s) d s .
\end{aligned}
$$

Remark that the solution of (5.4) is always well-defined in $X_{-1}$. In the Proposition 2.7 [17] it was proved that a classical solution of (5.3) which means that $x(\cdot)=x\left(\cdot, x_{0}, u\right)$ is continuously differentiable in $X$ and $x(t) \in \mathcal{D}\left(A_{m}\right)$ satisfying (5.1) is also a mild one. In order to obtain from (5.4) solutions having values in $X$ the authors impose some (regularity) sufficient condition on the elements in $\operatorname{ker}\left(\lambda-A_{m}\right)$ that is (a) $\operatorname{ker}\left(\lambda-A_{m}\right) \subseteq F_{A}$, or equivalently (b) $\operatorname{Range}\left(B_{\lambda}\right) \subseteq F_{A}$. 
Other equivalent conditions to (a) have been presented in [9].

It was been proved in Proposition 2.9 in [17] that under the assumption Range $\left(B_{\lambda}\right) \subseteq F_{A}$ and if in addition for some control $u \in U_{l o c}^{1}$ a classical solution $x(\cdot)$ of (5.3) exists, then this solution given by (5.5) below. As a classical solution of (5.3) associated to $x_{0} \in X$ and $u \in U_{l o c}^{1}$ is also a mild one, the following result shows that the above sufficient condition is even necessary for the existence of the mild solutions of (5.4) covering the Proposition 2.9 in [17].

Theorem 20. Let the initial state $x_{0} \in X$ and $\lambda \in \rho(A)$. Then system (5.1) admit a (unique) mild solution of all $u \in U_{l o c}^{1}$ if and only if Range $\left(B_{\lambda}\right) \subseteq F_{A}$. Moreover, for all $u \in U_{\text {loc }}^{1}$ its solution is given by

$$
x(t)=\mathbb{T}(t) x_{0}+(\lambda-A) \int_{0}^{t} \mathbb{T}(t-s) B_{\lambda} u(s) d s
$$

Proof. Sufficiency. Assume that Range $\left(B_{\lambda}\right) \subseteq F_{A}$ then by the closed graph theorem we obtain $B_{\lambda} \in \mathcal{L}\left(U, F_{A}\right)$ which implies $B^{\lambda}:=\left(\lambda I-A_{-1}\right) B_{\lambda} \in \mathcal{L}\left(U, F_{A_{-1}}\right)$. By virtue of Corollary 17 we obtain $B^{\lambda} \in \mathcal{A}_{1}(U, X, \mathbb{T})$, in particular $\int_{0}^{t} \mathbb{T}_{-1}(t-s) B^{\lambda} u(s) d s \in X$ for all $u \in U_{l o c}^{1}$. Hence system (5.3) has a unique mild solution satisfying

$$
x(t)=\mathbb{T}(t) x_{0}+(\lambda-A) \int_{0}^{t} \mathbb{T}(t-s) B_{\lambda} u(s) d s .
$$

accordingly to Lemma 2 .

Necessity. Assume that for all $u \in U_{l o c}^{1}$ system (5.3) has a mild solution denoted by $x(t)$. The fact that $x(t)$ is given by (5.4) we obtain

$$
\int_{0}^{t} \mathbb{T}_{-1}(t-s) B^{\lambda} u(s) d s \in X
$$

which implies that

$$
\left(\lambda-A_{-1}\right) B_{\lambda} \in \mathcal{A}_{1}(U, X, \mathbb{T}) .
$$

Thanks to Corollary 17 we obtain

$$
\text { Range }\left(\left(\lambda-A_{-1}\right) B_{\lambda}\right) \subseteq F_{A_{-1}} .
$$

Now, as $\left(\lambda I-A_{-1}\right)^{-1} \in \mathcal{L}\left(F_{A_{-1}}, F_{A}\right)$ we obtain Range $\left(B_{\lambda}\right) \subseteq F_{A}$. This end the proof. 


\section{Admissible Bilinear Control Operators and the Favard spaces}

In this section we come back to bilinear systems and show that the space of $(p, q)$ admissible control operators for bilinear systems is also related to the Favard spaces as in [16] where the conjugacy of $p, q$ (i.e. $q=p^{\prime}$ ) was considered.

Definition 21. Let $(\mathbb{T}(t))_{t \geq 0}, X$ and $V$ defined as in Definition 5 . We denote by $\mathcal{A}_{p, q}(X, V, \mathbb{T})$ the space of all $(p, q)$-admissible control operators for $(\mathbb{T}(t))_{t \geq 0}$. We endow this space with the norm

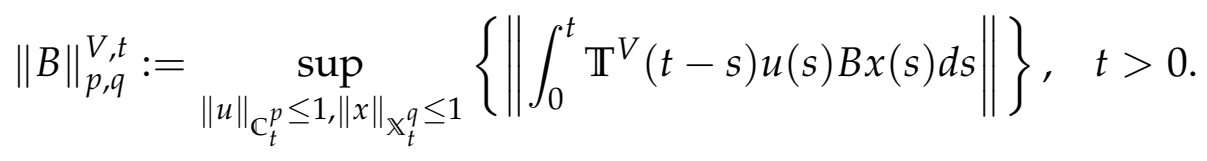

We would like to point out that we do not claim that the following Proposition is new, although it might be (it is only stated in [16] without proof under the conjugacy of $p$ and $q$ ).

Proposition 22. For all $(u, x)$ in $\mathbb{C}^{p} \times X^{q}$. Let $B \in \mathcal{A}_{p, q}(X, V, \mathbb{T})$, if we define $\Psi_{t}^{V}(\cdot, \cdot)$ for $t \geq 0$ by

$$
\Psi_{t}^{V}(u, x)=\int_{0}^{t} \mathbb{T}^{V}(t-s) u(s) B x(s)(s) d s
$$

then

$$
\|B\|_{p, q}^{V, t}=\left\|\Psi_{t}^{V}\right\|_{\mathcal{B L}}
$$

Proof. Using the property of causality property verified by the integral operator $\Psi_{t}^{V}$ we obtain

$$
\|B\|_{p, q}^{V, t}=\sup _{\|u\|_{\mathbb{C}_{t}^{p} \leq 1,\|x\|_{\mathbb{X}_{t}^{q}} \leq 1}}\left\{\left\|\mathbf{\Psi}_{t}\left(\underset{t}{u \diamond 0, x\rangle_{t} 0}\right)\right\|\right\}
$$

since $\|\underset{t}{u \diamond 0}\|_{\mathbb{C}^{p}}=\|u\|_{L^{p}(0, t)}$ and $\|\underset{t}{x \diamond 0}\|_{X^{q}}=\|x\|_{L^{q}(0, t ; X)}$ then $\|B\|_{p, q}^{V, t}=\left\|\Psi_{t}^{V}\right\|_{\mathcal{B} \mathcal{L}}$. [2].

In the sequel we need the following result which has been proved in [16] and

Proposition 23. In the conditions of Definition 21, the function $t \mapsto\left\|\Psi_{t}^{V}\right\|_{\mathcal{B L}}$ is monotonically increasing in $t$.

As for linear case the following result relies the space of $(p, q)$-admissible bilinear operators with some Favard spaces. This improves on earlier results in [16].

Proposition 24. In the conditions of Definition 5 such that $\mathcal{D}\left(A^{V}\right)=X$ we have

$$
\mathcal{L}\left(X, F_{A^{V}}\right) \hookrightarrow \mathcal{A}_{p, q}(X, V, \mathbb{T}) \hookrightarrow \mathcal{L}\left(X, F_{A^{V}}^{\alpha}\right),
$$

with $\alpha=\frac{1}{p}+\frac{1}{q}$. 
Before we give the proof, which is more or less along the lines of the linear case, let us comment on this proposition. If $B$ is $(p, q)$-admissible for $\left(\mathbb{T}(t)_{t \geq 0}\right)$, then it is easy to see that $B$ is $q$-admissible for $\left(\mathbb{T}(t)_{t \geq 0}\right)$, and that $\mathcal{A}_{p, q}(X, V, \mathbb{T}) \hookrightarrow$ $\mathcal{L}\left(X, F_{A^{V}}^{1 / q}\right)$ accordingly to Proposition 15.

Proof. (Proof of Proposition 24) The first injection: Let $B \in \mathcal{L}\left(X, F_{A^{V}}\right)$ and $(u, x) \in$ $\mathbb{C}_{l o c}^{p} \times X_{l o c}^{q}$. By using the generalized Hölder inequality we have $u \cdot x \in X_{l o c}^{1}$ then $u B x \in L_{\text {loc }}^{1}\left(\mathbb{R}^{+}, F_{A^{V}}\right)$. Hence by applying Lemma 2 (i) one obtains that $B \in \mathcal{A}_{p, q}(X, V, \mathbb{T})$. Since $\mathcal{D}\left(A^{V}\right)=X$, Lemma 2 (ii) and the generalized Hölder inequality yield:

$$
\begin{aligned}
\left\|\int_{0}^{t} \mathbb{T}^{V}(t-s) u(s) B x(s) d s\right\| & \left.\leq K\|u B x\|_{L^{1}\left(0, t ; F_{A} V\right.}\right) \\
& \leq K\|B\|_{\mathcal{L}\left(X, F_{A} V\right.}\|u\|_{\mathbb{C}_{t}^{p}}\|x\|_{\mathbb{X}_{t}^{q}} .
\end{aligned}
$$

Hence

$$
\left.\|B\|_{p, q}^{V, t} \leq K\|B\|_{\mathcal{L}\left(X, F_{A} V\right.}\right)
$$

The second injection: We argue in the same spirit as above: Let $B \in \mathcal{A}_{p, q}(X, V, \mathbb{T})$ since $F_{A^{V}}^{\alpha} \subseteq V$, it is enough by the closed graph theorem to show that Range $(B) \subseteq$ $F_{A^{V}}^{\alpha}$. So, let $x \in X, u_{\omega}(\cdot)=e^{-\omega \cdot}$ and $\xi_{x}(\cdot)=x$ on $\mathbb{R}^{+}$since

$$
e^{-\omega t} \mathbb{T}^{V}(t) B x-B x=\left(A^{V}-\omega I\right) \int_{0}^{t} e^{-\omega s} \mathbb{T}^{V}(s) B x d s
$$

taking $\omega \geq \max \left(0, \omega_{0}\left(\mathbb{T}^{V}\right)\right)$ and since $\mathcal{D}\left(A^{V}\right)=X$, we obtain

$$
\begin{aligned}
& \left\|e^{-\omega t} \mathbb{T}^{V}(t) B x-B x\right\|_{V} \leq K\left\|\int_{0}^{t} e^{-\omega s} \mathbb{T}^{V}(s) B x d s\right\| \\
& \leq K\left\|\Psi_{t}^{V}\left(u_{\omega}, \xi_{x}\right)\right\| \\
& \leq K \| \Psi_{t}^{V}\left(\underset{\omega}{\left.u_{\omega} \gg 0, \xi_{x} \gg 0\right)} \|\right. \\
& \leq K\left\|\Psi_{t}^{V}\right\|_{\mathcal{B L}} t^{\alpha}\|x\|
\end{aligned}
$$

then we have:

$$
\frac{1}{t^{\alpha}}\left\|e^{-\omega t} \mathbb{T}^{V}(t) B x-B x\right\|_{V} \leq K\left\|\Psi_{t}^{V}\right\|_{\mathcal{B} \mathcal{L}}\|x\|
$$

taking the supremum in $(0,1]$ and using Proposition 23 we obtain:

$$
\sup _{0<t \leq 1} \frac{1}{t^{\alpha}}\left\|e^{-\omega t} \mathbb{T}^{V}(t) B x-B x\right\|_{V} \leq K\left\|\Psi_{1}^{V}\right\|_{\mathcal{B L}}\|x\|
$$

then $B x \in F_{A^{V}}^{\alpha}$ implying that $B \in \mathcal{L}\left(X, F_{A^{V}}^{\alpha}\right)$.

Finally, by Proposition 22 we obtain $\|B\|_{\mathcal{L}\left(X, F_{A} V\right)} \leq K\|B\|_{p, q}^{V, 1}$. 
In the case of $q=p^{\prime}$ we rediscover the result in [16] saying that $\mathcal{A}_{p, p^{\prime}}(X, V, \mathbb{T})=\mathcal{L}\left(X, F_{A^{V}}\right)$.

When the underlying operator $A$ generates an analytic semigroup we have the following.

Proposition 25. Let $A$ generate a bounded analytic semigroup $(\mathbb{T}(t))_{t \geq 0}$ on a Banach space $X$, and let $\alpha=\frac{1}{p}+\frac{1}{q}$ and $\beta>0$ such that $\alpha<\beta \leq 1$ then

$$
\mathcal{L}\left(X, F_{A_{-1}}^{\beta}\right) \hookrightarrow \mathcal{A}_{p, q}(X, \mathbb{T}) \hookrightarrow \mathcal{L}\left(X, F_{A_{-1}}^{\alpha}\right) .
$$

Proof. We have only to prove the first injection. Let $B \in \mathcal{L}\left(X, F_{A_{-1}}^{\beta}\right)$ and $(u, x) \in$ $\mathbb{C}_{\text {loc }}^{p} \times X_{l o c}^{q}$ then

$$
\begin{aligned}
&\left\|\int_{0}^{t} \mathbb{T}_{-1}(t-s) u(s) B x(s) d s\right\| \\
& \quad=\left\|\int_{0}^{t}\left(-A_{-1}\right)^{-1}(t-s)^{1-\beta}\left(-A_{-1}\right) \mathbb{T}_{-1}(t-s) B x(s)(t-s)^{\beta-1} u(s) d s\right\| \\
& \quad \leq \int_{0}^{t}\left\|\left(-A_{-1}\right)^{-1}(t-s)^{1-\beta}\left(-A_{-1}\right) \mathbb{T}_{-1}(t-s) B x(s)(t-s)^{\beta-1} u(s)\right\| d s \\
& \quad=\int_{0}^{t}\left\|(t-s)^{1-\beta}\left(-A_{-1}\right) \mathbb{T}_{-1}(t-s) B x(s)(t-s)^{\beta-1} u(s)\right\|_{-1} d s .
\end{aligned}
$$

The fact that $\left(\mathbb{T}_{-1}(t)\right)_{t \geq 0}$ is a bounded analytic semigroup and that $\operatorname{Range}(B) \subset$ $F_{A_{-1}}^{\beta}$ we have

$$
\left\|(t-s)^{1-\beta}\left(-A_{-1}\right) \mathbb{T}_{-1}(t-s) B x(s)\right\|_{-1} \leq\|B\|_{\mathcal{L}\left(X, F_{A_{-1}}^{\beta}\right)}\|x(s)\|
$$

Thus

$$
\left\|\int_{0}^{t} \mathbb{T}_{-1}(t-s) u(s) B x(s) d s\right\| \leq\|B\|_{\mathcal{L}\left(X, F_{A_{-1}}^{\beta}\right)} \int_{0}^{t}(t-s)^{\beta-1}\|x(s)\||u(s)| d s
$$

and it is easy to verify with the generalized Hölder inequality and taking into account the condition on $\beta$ that the function $s \mapsto(t-s)^{\beta-1}\|x(s)\||u(s)|$ is $L_{l o c}^{1}\left(\mathbb{R}^{+}\right)$ then $B$ is $(p, q)$-admissible and there is $K:=K_{t}>0$ such that

$$
\|B\|_{p, q}^{X_{-1}, t} \leq K\|B\|_{\mathcal{L}\left(X, F_{A_{-1}}^{\beta}\right)}
$$

The following result covers the result in [2] and it is certainly more general than this ones where the author needs $\gamma=1 / 2$ and it will suffice for the application we have in mind.

Theorem 26. Let A generate a bounded analytic semigroup $(\mathbb{T}(t))_{t \geq 0}$ on a Banach space $X$, and $p, q \in] 1,+\infty\left[\right.$ satisfying (1.4). Then the fractional power $(-A)^{\gamma}$ is $(p, q)$ admissible for all $\gamma \in(0,1)$ with $\frac{1}{p}+\frac{1}{q}<1-\gamma$. 
Proof. Let $(u, x) \in \mathbb{C}_{l o c}^{p} \times X_{l o c}^{q}$. The fact that $\mathcal{D}\left(A_{-1}\right)=X \subset \mathcal{D}\left(\left(-A_{-1}\right)^{\gamma}\right)$ and that $\left(-A_{-1}\right)^{\gamma} \in \mathcal{L}\left(X, X_{-1}\right)$ (see, Theorem 5.34 [11]) the $X_{-1}$-valued integral

$$
\Psi_{t}(u, x)=\int_{0}^{t} \mathbb{T}_{-1}(t-s) u(s)\left(-A_{-1}\right)^{\gamma} x(s) d s
$$

is well-defined. Since $(\mathbb{T}(t))_{t \geq 0}$ is an analytic semigroup $\left(\mathbb{T}_{-1}(t)\right)_{t \geq 0}$ is so, this implies that $\mathbb{T}_{-1}(t-s)\left(-A_{-1}\right)^{\gamma} x(s) \in X$ and there exists $K:=K_{\gamma}$ such that

$$
\begin{aligned}
\left\|\mathbb{T}_{-1}(t-s)\left(-A_{-1}\right)^{\gamma} x(s)\right\| & =\left\|(-A)^{\gamma} \mathbb{T}(t-s) x(s)\right\| \\
& \leq K_{\gamma}(t-s)^{-\gamma}\|x(s)\| .
\end{aligned}
$$

It follows that

$$
\begin{aligned}
\left\|\Psi_{t}(u, x)\right\| & \leq \int_{0}^{t}\left\|\mathbb{T}_{-1}(t-s) u(s)\left(-A_{-1}\right)^{\gamma} x(s)\right\| d s \\
& \leq K_{\gamma} \int_{0}^{t}(t-s)^{-\gamma}\|x(s)\||u(s)| d s .
\end{aligned}
$$

Since $\frac{1}{p}+\frac{1}{q}<1-\gamma$ it easy to check that the function $s \mapsto(t-s)^{-\gamma}\|x(s)\||u(s)|$ is $\mathbb{C}_{\text {loc }}^{1}$ and there is a constant $K:=K_{t}>0$ such that

$$
\left\|\Psi_{t}(u, x)\right\| \leq K\|u\|_{\mathbb{C}_{t}^{p}}\|x\|_{\mathbb{X}_{t}^{q}}
$$

which ends the proof.

\section{Example}

In this section we apply the obtained results to fractional equation of diffusion type.

Let $\Omega$ be an open bounded domain in $\mathbb{R}^{n}$ with sufficiently smooth boundary $\Gamma$. We study a time-fractional diffusion equation of distributed order of the form

$$
\begin{cases}\frac{\partial y}{\partial t}=\Delta y+u(t)(-\Delta)^{\gamma} y & \text { on }(0,+\infty) \times \Omega, \\ y=0 & \text { on }(0,+\infty) \times \Gamma \\ y(0, x)=y_{0}(x) & \text { on } \Omega,\end{cases}
$$

where $\gamma \in] 0,1]$.

Recall that fractional Laplacian $(-\Delta)^{\gamma}$ as long as $\gamma>1 / 2$ is widely used to describe physical systems exhibiting anomalous diffusions (see. e.g. [19], [20], [15]). The system has the form (1.1) if we set $X=L^{2}(\Omega), B=(-\Delta)^{\gamma}$ and

$$
\mathcal{D}(A)=H^{2}(\Omega) \cap H_{0}^{1}(\Omega), \quad A y=\Delta y .
$$

Let $0<\beta_{1}<\beta_{2}<\cdots<\beta_{n}<\cdots$ be the set of eigenvalues of $-A$ and denote by $\left\{\varphi_{n}\right\}_{n}$ the corresponding orthonormal basis in $L^{2}(\Omega)$. It is well-known that $A$ generates an analytic semigroups $\mathbb{T}(t)$ which is given explicitly by

$$
\mathbb{T}(t) y=\sum_{n}\left\langle y, \varphi_{n}\right\rangle e^{-\beta_{n} t} \varphi_{n} \quad \text { for all } y \in L^{2}(\Omega),
$$


where $\langle\cdot, \cdot\rangle$ denotes the scalar product in $L^{2}(\Omega)$. Moreover, the operator $B$ can be expressed by

$$
B y=\sum_{n}\left\langle y, \varphi_{n}\right\rangle \beta_{n}^{\gamma} \varphi_{n}
$$

Clearly $B$ is unbounded on $L^{2}(\Omega)$ and it is bounded from $L^{2}(\Omega)$ onto the space $V$ defined as the completion of $L^{2}(\Omega)$ for the norm defined by

$$
\|y\|_{V}^{2}=\sum_{n} \frac{\left\langle y, \varphi_{n}\right\rangle^{2}}{\beta_{n}^{2 \gamma}} .
$$

Recall that the space $\mathcal{D}\left((-A)^{\gamma}\right)$ is normed

$$
\|y\|_{\mathcal{D}\left((-A)^{\gamma}\right)}^{2}=\sum_{n} \beta_{n}^{2 \gamma}\left(\left\langle y, \varphi_{n}\right\rangle\right)^{2}
$$

It is easy to see that the space $V$ can be interpreted as the dual space of $\mathcal{D}\left((-A)^{\gamma}\right)$ with respect to the $L^{2}(\Omega)$-topology, the space $L^{2}(\Omega)$ being the pivot space. Furthermore, the the extension (respectively the restriction) of the operator $A$ to $V$ (respectively $\mathcal{D}\left((-A)^{\gamma}\right)$ ) generates a strongly continuous semigroup denoted by $\left(\mathbb{T}^{V}(t)\right)_{t \geq 0}$ (see. [1], Vol.1).

Thanks to Theorem 26, we obtain the following condition which extends the result given in [2] where the author needs $\gamma=1 / 2$.

Proposition 27. For all $\gamma \in] 0,1[$ and $p, q \in] 1,+\infty[$ satisfying

$$
\frac{1}{p}+\frac{1}{q}<1-\gamma
$$

the operator $(-\Delta)^{\gamma}$ is $(p, q)$-admissible w.r.t $X_{-1}$ and $V$.

Proof. The $(p, q)$-admissibility w.r.t to $X_{-1}$ is given by Theorem 26 and hence the $(p, q)$-admissibility w.r.t to $V$ can be trivially obtained by similar arguments after having replaced $X_{-1}$ by $V$ due to analyticity of $\left(\mathbb{T}^{V}(t)\right)_{t \geq 0}$ and that $B$ is simply the restriction of $\left(-A^{V}\right)^{\gamma}$ to $X$.

Remark 28. Clearly for all $\gamma \in] 0,1]$ the control operator $(-\Delta)^{\gamma}$ is unbounded on $L^{2}(\Omega)$. Hence $(-\Delta)^{\gamma}$ cannot be $\left(p, p^{\prime}\right)$-admissible w.r.t to $X_{-1}$ and $V$ accordingly to Corollary 4.8 [16] and it was already proved in [2] by a (quantitative) manner for $\gamma=1 / 2$ w.r.t $V$.

Finally, combining Proposition 27 and Theorem 2.5 [2] leads to the following corollary.

Corollary 29. Let $\gamma \in] 0,1[$ and $p \in] \frac{1}{1-\gamma},+\infty\left[\right.$. Then for any $y_{0} \in L^{2}(\Omega)$ and $u \in \mathbb{C}_{\text {loc, }}^{p}$ the system (7.1) admits a unique mild solution $y(t)$ on $\mathbb{R}^{+}$. 


\section{Conclusion}

In this work, we have considered a class of linear and bilinear control systems which allow some unboundedness of control operators. Links between the notion of $p$-admissibility for linear systems (resp. $(p, q)$-admissibility for bilinear systems) and Favard class are given. In particular and concerning linear systems, we have identified the space of 1-admissible control operator for a given linear system which constitutes a generalization of the result in Theorem [29], more we have shown that the Weiss-conjecture holds for $p=1$ in general Banach setting. Based on this, an application to boundary control systems is given. More, the authors in [6] have shown that every well-posed linear system in $L^{1}$ is weakly regular, if the adjoint of its associated $C_{0}$-semigroup is also a $C_{0}$-semigroup. (see. [27] for more information about well-posed linear systems). Regular bilinear systems which has been introduced in [3] constitutes an important subclass of the wellposed bilinear systems, see [3]. So, it has been proved in [6] that every well-posed bilinear system as introduced in [3] is weakly regular under the same assumption on its underlying semigroups. Finally, it would be interesting to study bilinear systems with delays in state and control. This may give rise to another class of bilinear systems. That will be the subject of our future work.

\section{References}

[1] Da Prato G. Delfour M.C Bensoussan, A. and S.K Mitter. Representation and control of infinite dimensional systems,Vol. 1, 2. Birkhäuser Boston, 1993.

[2] L. Berrahmoune. A note on admissibility for unbounded bilinear control systems. Bulletin of the Belgian Mathematical Society-Simon Stevin, 16:193-204, 2009.

[3] H. Bounit and A. Idrissi. Regular bilinear systems. IMA Journal of Mathematical Control and Information, 22:26-57, 2005.

[4] H. Bounit, A. Driouich and O. El-Mennaoui. A direct approach to the Weiss conjecture for bounded analytic semigroups. Czechoslovak Mathematical Journal, 60:527-539, 2010.

[5] H. Bounit, A. Driouich and O. El-Mennaoui. A direct approach to the weighted admissibility of observation operators for bounded analytic semigroups. Semigroup Forum, 83:52-64, 2011.

[6] H. Bounit and F. Maragh. On the regularity for some class of $L^{p}$-well-posed linear systems, Transnational Journal of Mathematical Analysis and Applications, 2, 37-65, 2014.

[7] P.L. Butzer and H. Berens. Semi-Groups of Operators and Approximation. Springer-Verlag Berlin, 1967.

[8] R. F. Curtain. The Salamon-Weiss class of well-posed infinite-dimensional linear systems: a survey IMA Journal of Mathematical Control and Information, 2:207-223, 1997 
[9] W. Desch and W. Schappacher. Some generation results for perturbed semigroups Trends in semigroup theory and applications, Ph. Clement et al (Eds.), Marcel Dekker, New York, 116:125-152, 1989.

[10] Z. Emirsjlow and S. Townley. From PDEs with boundary control to the abstract state equation with an unbounded input operator: a tutorial. European Journal of Control, 6:27-49, 2000.

[11] K.-J. Engel and R. Nagel. One-parameter semigroups for linear evolution equations. Springer Verlag, 2000.

[12] G. Greiner. Perturbing the boundary conditions of generator. Houston Journal of mathematics, 13:213-229, 1987.

[13] B.H. Haak and P.C. Kunstmann. Weighted admissibility and wellposedness of linear systems in Banach spaces. SIAM Journal Control and Optimization, 45:2094-2118, 2007.

[14] B. Jacob H. Zwart and O. Staffans. Weak admissibility does not imply admissibility for analytic semigroups. Systems and Control Letters, 48:341-350, 2003.

[15] J. Klafter I. Sokolov and A. Blumen. Fractional kinetics. Physics Today, 55: 48-54, 2002.

[16] A. Idrissi. On the unboundedness of control operators for bilinear systems. Quaestiones Mathematicae, 7:105-123, 2003.

[17] B. Klöss R. Nagel K.-J. Engel, M.K. Fijavz and E. Sikolya. Maximal controllability for boundary control. Applied Mathematics E Optimization, 62:205-227, 2010.

[18] C. Le Merdy. The weiss conjecture for bounded analytic semigroups. Journal of the London Mathematical Society, 67(3)(3):715-738, 2003.

[19] R. Metzler and J. Klafter. The random walks guide to anomalous diffusion: a fractional dynamic approach. Physics Reports, 339:1-77, 2000.

[20] R. Metzler and J. Klafter. The restaurant at the end of the random walk: recent developments in the description of anomalous transport by fractional dynamics. Journal of Physics A: Mathematical and General, 37:161-208, 2004.

[21] R. Nagel. Sobolev spaces and semigroups Semesterbericht Funktionalanalysis, 4:1-20, 1983.

[22] R. Nagel and E. Sinestrari. Inhomogeneous Volterra integrodifferential equations for Hille-Yoshida operators. Lecture Notes in Pure and Applied Mathematics, 47:150, 1994.

[23] J.M.A.M. Van Neerven. The Adjoint of a Semigroup of Linear Operators, Lecture Notes Math. Springer-Verlag, Berlin, 1992. 
[24] G. Da Prato and P. Grisvard. Maximal regularity for evolution equations by interpolation and extrapolation. Journal of functional analysis, 58:107-124, 1984.

[25] D. Salamon. Infinite-dimensional linear system with unbounded control and observation: a functional analytic approach. Transactions of American Mathematical Society, 300:383-431, 1987.

[26] O. Staffans and G. Weiss. Well-posed linear systems in $L^{1}$ and $L^{\infty}$ are regular. in Proceedings of MMAR'98, Methods and Models in Automation and Control, 25-29 August, Miedzyzdroje, Poland, 75-80, 1998.

[27] O. Staffans. Well-Posed Linear Systems. Cambridge university press, 2005.

[28] Michael Unteregge. $p$-admissible control elements for diagonal semigroups on $l^{r}$-spaces. Systems and Control Letters, 56(6):447-451, 2007.

[29] G. Weiss. Admissibility of unbounded control operators. SIAM Journal on Control and Optimization, 27:527-545, 1989.

[30] G. Weiss. Two conjectures on the admissibility of control operators. Birkhäuser Verlag, Basel, In F. Kappel, W. Desch, editors, Estimation and Control of Distributed Parameter Systems:367-378, 1989.

Faculty of Sciences;

Laboratory of Applied Mathematics and Applications (Functional Analysis Group). BP. 8106, Cité Dakhla, Agadir Maroc.

Corresponding author, e-mail: h.bounit@uiz.ac.ma.

Université Lyon 1; CNRS UMR 5007;

Laboratory of Process Control and Chemical Engineering (LAGEP),

43 bd du 11 novembre,

69100 Villeurbanne, France.

e-mail: hammouri@lagep.univ-lyon1.fr. 